
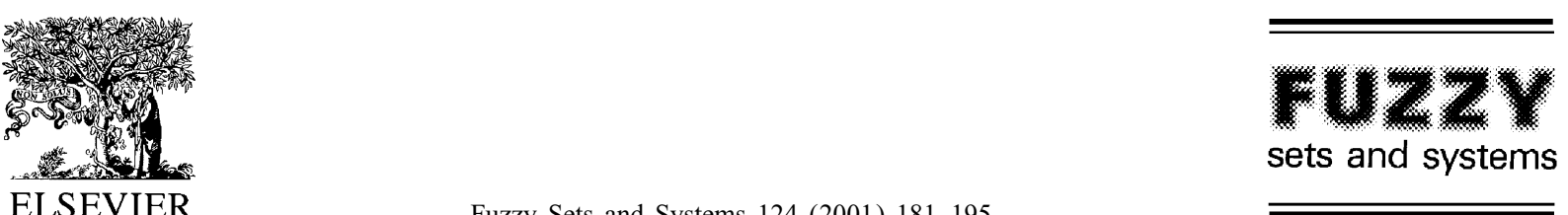

Fuzzy Sets and Systems 124 (2001) 181-195

www.elsevier.com/locate/fss

\title{
Robust neuro-fuzzy control of multivariable systems by tuning consequent membership functions
}

\author{
Wei-Song Lin ${ }^{\mathrm{a}, *}$, Chih-Hsin Tsai ${ }^{\mathrm{a}}$, Jing-Sin Liu ${ }^{\mathrm{b}}$ \\ a Department of Electrical Engineering, National Taiwan University, No. 1, Sec. 4, Roosevelt Rd., Taipei 106, Taiwan, ROC \\ ${ }^{\mathrm{b}}$ Institute of Information Science, Academia Sinica, Nankang, Taipei, Taiwan 115, ROC
}

Received 10 August 1998; received in revised form 8 October 1999; accepted 6 April 2000

\begin{abstract}
A robust neuro-fuzzy controller with tuning mechanism of membership functions and neural weights to achieve the tracking control of composite multivariable systems is proposed. The control strategy is developed to facilitate robust property by self-tuning the consequent membership functions of the fuzzy controllers. By an on-line tuning mechanism, the fuzzy system can effectively deal with the equivalent uncertainties that may appear in the subsystems due to plant uncertainty, function approximation error, or external disturbance. By using Lyapunov stability theory, the overall system with the proposed controller has been proved to be uniform ultimate bounded. Simulation results of a two-link robot control demonstrate the effectiveness and robustness of the design. (C) 2001 Elsevier Science B.V. All rights reserved.
\end{abstract}

Keywords: Neuro-fuzzy control; Consequence membership function; Multivariable systems

\section{Introduction}

The fuzzy control method has been demonstrated to have advantage of robustness through industrial applications $[9,11]$ and theoretical analysis $[4,6,19]$. In [4], a robustness measurement, which gives the bound on allowable uncertainties or nonlinearity, and robust stability of fuzzy control systems have been studied through the Popov-Lyapunov approach. However, the control system to be analyzed should be able to transform into a perturbed Lur'e system. Yi and Chung [19] presented control theoretic analysis of a fuzzy control system in the sense of Lyapunov based on the similarity between prevalent fuzzy logic controllers and the variable structure controller. In [6], Johansen used fuzzy sets and fuzzy inference to construct a nonlinear model of plant and provided an analysis of stability, robustness and performance of the control loop. The controller is a discrete-time nonlinear decoupler but nonfuzzy model based.

\footnotetext{
* Corresponding author. Tel.: +886-2-2363-5251 ext. 413; fax: +886-2-2363-8247.

E-mail address: weisong@cc.ee.ntu.edu.tw (W.-S. Lin).
} 
Recently, using the fuzzy basis function expansion (FBFE) to represent the unknown nonlinearity of plants, several researchers have proposed adaptive fuzzy control methods [7,13,17,20]. These methods take the advantages of fuzzy basis function and stable parameter adaptation schemes and are derived by using Lyapunov theory. However, as indicated in [8,16], the adaptive fuzzy controllers which will change their parameters of membership function of fuzzy rules used in fuzzy inference may result in ineffectualness of the robust property of fuzzy control. In [2], by adding a robust stability factor to the controller, an adaptive law is obtained to be robust with respect to the modeling error resulting from the fuzzy approximators. Here we propose a robust neuro-fuzzy controller with self-tuning on the consequent membership function to encounter the equivalent uncertainty resulted by function approximation error, external disturbances, and measurement noise. But no additional robust stability factor as in [2] is added in the design since it will make the entire controller not being in a context of fuzzy logic control system. The robustness of the neuro-fuzzy control system against system uncertainties is analyzed; this analysis gives an account of the relationship between control performance and the design parameters of the neuro-fuzzy controller, which was previously obscure in the theory of fuzzy/neural control. Comparing with the results in $[12,14,17]$, our effort has been on the extension to composite multivariable systems and the robust parameter adaptation schemes. Two weight adaptation schemes are proposed and compared. One is the gradient weight scheme that is widely used in adaptive fuzzy/neural control law $[10,13,17]$. Another is a robust adaptation scheme with self-tuning on the consequent membership functions of the fuzzy part. The result of the former approach can only be proved when the parameters are initialized not too far from their optimal values (local stability) [10]. The latter we proposed is shown to release the above assumptions and be able to considerably reduce the tracking error residual set and obtain robustness in the sense that the self-tuning mechanism can automatically adapt the controller to react to the effect of equivalent uncertainties for unknown plant dynamics. The overall system with the adaptation schemes has been proved to be able to guarantee uniform ultimate bounded.

The remainder of this paper is organized as follows: The output-tracking problem of composite multivariable nonlinear systems is formulated in Section 2. The robust neuro-fuzzy controller for multivariable systems is proposed and formulated in Section 3. The gradient adaptation and the robust adaptation schemes are proposed and compared in Section 4. In Section 5, the control of a two-link robot carrying a heavy load is simulated to illustrate the effectiveness and robustness of the proposed control system. Section 6 is the conclusion.

\section{Problem formulation}

Consider a composite multivariable nonlinear plant governed by

$$
\boldsymbol{y}^{(r)}=\boldsymbol{f}(\boldsymbol{x})+\boldsymbol{G}(\boldsymbol{x}) \boldsymbol{u}+\boldsymbol{d}(\boldsymbol{x}, t),
$$

where $\boldsymbol{y}=\left[y_{1}, \ldots, y_{m}\right]^{\mathrm{T}}$ and $\boldsymbol{y}^{(r)} \equiv\left[y_{1}^{\left(r_{1}\right)}, \ldots, y_{m}^{\left(r_{m}\right)}\right]^{\mathrm{T}}$ denote the output vector and its derivative, respectively, $r=\left[r_{1}, \ldots, r_{m}\right]$ with $\sum_{i=1}^{m} r_{i}=n$ is defined as the system relative degree, $\boldsymbol{u}=\left[u_{1}, \ldots, u_{m}\right]^{\mathrm{T}}$ is the system input, $\boldsymbol{x}=\left[x_{1}, \ldots, x_{n}\right]^{\mathrm{T}}=\left[y_{1}, \ldots, y_{1}^{\left(r_{1}-1\right)}, \ldots, y_{m}, \ldots, y_{m}^{\left(r_{m}-1\right)}\right]^{\mathrm{T}}$ is the state vector, $\boldsymbol{f}(\boldsymbol{x})=\left[f_{1}(\boldsymbol{x}), \ldots, f_{m}(\boldsymbol{x})\right]^{\mathrm{T}}, \boldsymbol{G}(\boldsymbol{x})$ $=\left[\boldsymbol{g}_{1}(x), \ldots, \boldsymbol{g}_{m}(\boldsymbol{x})\right], f_{i}(\boldsymbol{x})$ and $\boldsymbol{g}_{i}(\boldsymbol{x})=\left[g_{i 1}(\boldsymbol{x}), \ldots, g_{i m}(\boldsymbol{x})\right]^{\mathrm{T}}$ are unknown smooth functions satisfying the mild assumption $\left|g_{i j}(\boldsymbol{x})\right|>0, \forall \boldsymbol{x}$, and $\boldsymbol{d}(\boldsymbol{x}, t)=\left[d_{1}(\boldsymbol{x}, t), \ldots, d_{m}(\boldsymbol{x}, t)\right]$ is the disturbance with the properties of standard smoothness and boundedness. Then given a desired trajectory $\boldsymbol{y}_{\mathrm{d}}(t)$, the purpose of design is to find a controller for the composite multivariable plant described by (1) so that the tracking error represented by $\boldsymbol{e}=\left[\boldsymbol{e}_{1}^{\mathrm{T}}, \ldots, \boldsymbol{e}_{m}^{\mathrm{T}}\right]^{\mathrm{T}}$ with $\boldsymbol{e}_{i}=\left[y_{i}-y_{i \mathrm{~d}}, \dot{y}-\dot{y}_{i \mathrm{~d}}, \ldots, y_{i}^{\left(r_{i}-1\right)}-y_{i \mathrm{~d}}^{\left(r_{i}-1\right)}\right]^{\mathrm{T}}$ will be attenuated to an arbitrarily small residual tracking error set. 


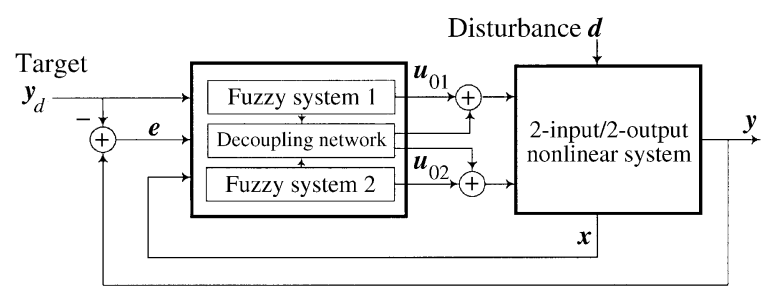

Fig. 1. Configuration of a robust neuro-fuzzy control system.

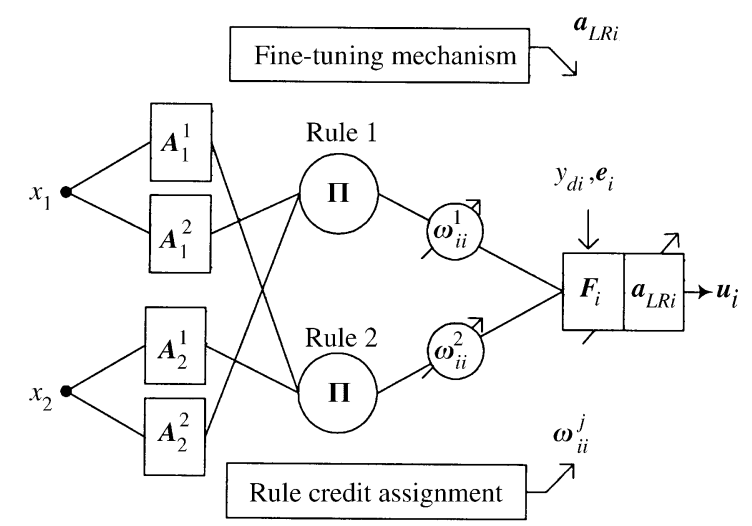

Fig. 2. The synaptic connection topology of the $i$ th multi-layer fuzzy system.

\section{Design of the robust neuro-fuzzy controller}

The control of an MIMO nonlinear system poses difficulties mainly in three aspects. Firstly, the interactions among subsystems usually cause the input applied to one subsystem undesirably affecting some other subsystems. Secondly, the functions $\boldsymbol{f}$ and $\boldsymbol{G}$ or parameters of the system are being unknown or difficult to measure. The final one is the presence of equivalent uncertainties which resulted from the function approximation error and the disturbances. To overcome the above difficulties and shrink the tracking error residual set, the proposed robust neuro-fuzzy controller is composed of the following three parts: a multi-layer fuzzy system with rule credit assignment, a self-tuning mechanism on the consequent membership functions, and a decoupling network. The configuration of the robust neuro-fuzzy control system in the case of controlling two-input/two-output nonlinear system is shown in Fig. 1. The multi-layer fuzzy system and the decoupling network are nominal designs based on an on-line approximation of the unknown nonlinear functions of the plant. The self-tuning mechanism is designed to encounter the equivalent uncertainty which resulted from the plant uncertainty, the function approximation error, or the external disturbances.

\subsection{The multi-layer fuzzy system}

Fig. 2 shows the proposed synaptic connection topology of the $i$ th multi-layer fuzzy system for the $i$ th subsystem of the controlled plant. Considering the request of numerical input and output of the fuzzy system, a particular class of fuzzy system with the singleton fuzzified, algebraic product T-norm, the sup star compositional operator [17] and the local mean-of-maximum method [1] are used. The basic components of the multi-layer fuzzy system and its self-tuning mechanism will be discussed and formulated in the following paragraph.

Fuzzy rule base: A multivariable system can be controlled by the following linguistic rules

$$
\begin{aligned}
& R^{j}: I F x_{1} \text { is } A_{1}^{j} A N D \cdots A N D x_{n} \text { is } A_{n}^{j} \\
& \text { THEN } u_{1} \text { is } B_{1}^{j} \cdots u_{m} \text { is } B_{m}^{j}, \quad j=1, \ldots, N+1,
\end{aligned}
$$

where $N+1$ is the number of fuzzy rules, the antecedent part, $A_{k}^{j}$, is defined as the following Gaussian type:

$$
A_{k}^{j}\left(x_{k}\right)=\exp \left(-\left(x_{k}-m_{k}^{j}\right)^{2} / a_{k}^{j}\right)
$$


and the consequent membership function of the consequent part is defined as

$$
B_{i}^{j}\left(u_{i}\right)= \begin{cases}\left(1+\left(\left(c_{i}^{j}-u_{i}\right) / a_{\mathrm{L} i}\right)^{2}\right)^{-1} & \text { if } u_{i} \leqslant c_{i}^{j}, \\ \left(1+\left(\left(u_{i}-c_{i}^{j}\right) / a_{\mathrm{R} i}\right)^{2}\right)^{-1} & \text { if } u_{i}>c_{i}^{j},\end{cases}
$$

where $\left\{a_{k}^{j}, m_{k}^{j}\right\}$ and $\left\{a_{\mathrm{L} i}, a_{\mathrm{R} i}, c_{i}^{j}\right\}$ are referred to the premise and consequence parameters, respectively.

Rule credit assignment: The basic idea of the rule credit assignment is to reward good rules by increasing the confidence of the consequent fuzzy sets and the recommendation fuzzy output of this rule. Denote $\omega_{i i}^{j}>1$ (or $\omega_{i i}^{j}<1$ ) as a reward (or a punishment) offered to the $j$ th rule in the $i$ th knowledge rule base, then the consequent membership function (3) can be reshaped into

$$
\tilde{B}_{i}^{j}\left(u_{i}\right)= \begin{cases}\left(1+\left(\omega_{i i}^{j}\left(c_{i}^{j}-u_{i}\right) / a_{\mathrm{L} i}\right)^{2}\right)^{-1} & \text { if } u_{i} \leqslant c_{i}^{j}, \\ \left(1+\left(\omega_{i i}^{j}\left(u_{i}-c_{i}^{j}\right) / a_{\mathrm{R} i}\right)^{2}\right)^{-1} & \text { if } u_{i}>c_{i}^{j}\end{cases}
$$

and the recommendation fuzzy output of each rule is determined in singleton form as follows:

$$
\omega_{i i}^{j} I\left(\boldsymbol{A}^{j}\left(\boldsymbol{x}^{0}\right), \tilde{B}_{i}^{j}\left(u_{i}\right)\right)= \begin{cases}\omega_{i i}^{j} \boldsymbol{A}^{j}\left(\boldsymbol{x}^{0}\right) & \text { for } u_{i}=\tilde{c}_{i}^{j}, \\ 0 & \text { otherwise },\end{cases}
$$

where $\boldsymbol{x}^{0}=\left(x_{1}^{0}, \ldots, x_{n}^{0}\right), \boldsymbol{A}^{j}\left(\boldsymbol{x}^{0}\right)=A_{1}^{j}\left(x_{1}^{0}\right) A_{2}^{j}\left(x_{2}^{0}\right) \cdots A_{n}^{j}\left(x_{n}^{0}\right)$ denotes the given input and the matching degree, respectively, $\tilde{c}_{i}^{j}$ denotes the location of the singleton implication fuzzy set and is defined as (see Fig. 5 [1])

$$
\tilde{c}_{i}^{j}=\text { the centroid of the set }\left\{u_{i}: \tilde{B}_{i}^{j}\left(u_{i}\right) \geqslant \boldsymbol{A}^{j}\left(\boldsymbol{x}^{0}\right)\right\} .
$$

Using (4) and (6) can be resolved into (see Appendix A)

$$
\tilde{c}_{i}^{j}=c_{i}^{j}-\frac{a_{\mathrm{LR} i}}{\omega_{i i}^{j}} \sqrt{\frac{1}{\boldsymbol{A}^{j}}-1}
$$

where $a_{\mathrm{LR} i}=\left(a_{\mathrm{L} i}-a_{\mathrm{R} i}\right) / 2$.

The study of assigning rule credit assignment may be complicated, where, the modification of control rules is achieved by giving a credit or reward value to individual rules engaged in the problem solving process. In $[15,18]$, the credit value is obtained from a fuzzy algorithm which defines the desired performance linguistically. However, as addressed in $[3,21]$, the control rules may often be improperly modified when the set-point changes. Generally, for a fuzzy/neural system, these parameters are updated according to the output value and the associated teacher signals. But for the control problems under consideration, the teacher signals are not available and only the error information between the plant and the desired trajectory can be used. Therefore, in this paper, the entire problem is approached in the context of Lyapunov-based adaptive systems theory to provide on-line tuning rules for $\omega_{i i}^{j}$ as shown in the next section.

Self-tuning mechanism: Physically, the parameter $a_{\mathrm{LR} i}$ represents the left-right spread difference of the consequent membership functions. In traditional fuzzy logic control system, $a_{\mathrm{L} i}$ is set to be equivalent to $a_{\mathrm{R} i}$ or the consequent membership is just in singleton form [13]. In this paper, this term is employed as a robust control component and a robust adaptive law for it is proposed in the next section.

Analytical formulation of the multi-layer fuzzy system: Using the center average defuzzification, the output response of the fuzzy controller is

$$
u_{0 i}=F_{i}\left(\boldsymbol{A}^{j}, \omega_{i i}^{j}\right)=\frac{\sum_{j=1}^{N+1} \omega_{i i}^{j} \boldsymbol{A}^{j} \tilde{c}_{i}^{j}}{\sum_{j=1}^{N+1} \omega_{i i}^{j} \boldsymbol{A}^{j}} .
$$


In the rule base, the $(N+1)$ th rule is chosen to be of Takagi-Sugeno type and its consequent membership function $B_{i}^{N+1}$ is singleton with support represented as the form of the synthesis input

$$
c_{i}^{\prime}=y_{\mathrm{d} i}^{\left(r_{i}\right)}-\alpha_{i}^{\mathrm{T}} e_{i}
$$

where $\alpha_{i}=\left[\alpha_{i 1}, \ldots, \alpha_{i r_{i}}\right]^{\mathrm{T}}$ being positive constants chosen such that $p^{n}+\alpha_{i r_{i}} p^{r_{i}-1}+\cdots+\alpha_{i 1}$ is a stable (Hurwitz) polynomial. The curvature control parameter of its antecedent membership function, $a_{k}^{N+1}$, is assumed to approach to infinity so that this rule will be fired whatever $\boldsymbol{x}^{0}$ is. The credit assignment takes place in rules $R^{j}, j=1, \ldots, N$ but assigned to be 1 for $R^{N+1}$. Accordingly, using (7) and (9), the analytical formulation of the multi-layer fuzzy system in Eq. (8) resolves into

$$
\boldsymbol{u}_{0}=\hat{\boldsymbol{D}}^{-1}\left(-\hat{\boldsymbol{f}}+\boldsymbol{c}^{\prime}-\boldsymbol{a}_{\mathrm{LR}}^{\mathrm{T}} \hat{f}_{\mathrm{LR}}\right),
$$

where $\hat{\boldsymbol{D}}=$ Block $\operatorname{diag}\left[\boldsymbol{\omega}_{11}^{\mathrm{T}} \hat{\boldsymbol{g}}_{\omega}, \ldots, \boldsymbol{\omega}_{m m}^{\mathrm{T}} \hat{\boldsymbol{g}}_{\omega}\right], \hat{\boldsymbol{f}}=\left[\boldsymbol{\theta}_{1}^{\prime \mathrm{T}} \hat{\boldsymbol{f}}_{\theta}, \ldots, \boldsymbol{\theta}_{m}^{\prime \mathrm{T}} \hat{\boldsymbol{f}}_{\theta}\right]^{\mathrm{T}}$ with $\boldsymbol{\omega}_{i i}$ and $\hat{\boldsymbol{g}}_{\omega}$ are $(N+1) \times 1$ column vectors composed of $\omega_{i i}^{j}$ and $\boldsymbol{A}^{j}$, respectively, $\boldsymbol{\theta}_{i}^{\prime}$ and $\hat{\boldsymbol{f}}_{\theta}$ are $N \times 1$ column vectors composed of $\omega_{i i}^{j} c_{i}^{j}$ and $-\boldsymbol{A}^{j}$, respectively, $\boldsymbol{c}^{\prime}=\left[c_{1}^{\prime}, \ldots, c_{m}^{\prime}\right]^{\mathrm{T}}, \boldsymbol{a}_{\mathrm{LR}}=\left[a_{\mathrm{LR} 1}, \ldots, a_{\mathrm{LR} m}\right]^{\mathrm{T}}$ and $\hat{f}_{\mathrm{LR}}=\sum_{j=1}^{N} \boldsymbol{A}^{j} \sqrt{1 / \boldsymbol{A}^{j}-1}$.

\subsection{The decoupling neural network and the overall control law}

Since the multi-layer fuzzy system does not take the interconnection among subsystems into consideration, the decoupling network is required to eliminate the interaction. The construction of the decoupling neural network is conceptually explained as below. Let $\boldsymbol{D}(\boldsymbol{x})=$ Block $\operatorname{diag}\left[g_{11}, \ldots, g_{m m}\right]$ and assuming that the subsystems are not interconnected (i.e., $g_{i j}=0, \forall i \neq j$ ), and the nonlinear function $\boldsymbol{f}(\boldsymbol{x})$ of the system is known exactly, then the desired control input $\boldsymbol{u}_{0}^{*}$ for each individual subsystem without disturbance can be theoretically computed from

$$
\boldsymbol{u}_{0}^{*}=\boldsymbol{D}^{-1}\left(-\boldsymbol{f}+\boldsymbol{c}^{\prime}\right)
$$

where $\boldsymbol{\alpha}_{i}, i=1, \ldots, m$ are chosen to obtain exponential decay of the tracking error. When the subsystems are interconnected (i.e. $g_{i j} \neq 0$ ), to ensure $\boldsymbol{e} \rightarrow 0$ as $t \rightarrow \infty$, the desired control input needs to counteract the interactions as

$$
\begin{aligned}
\boldsymbol{u}^{*} & =\boldsymbol{G}^{-1}\left(-\boldsymbol{f}+\boldsymbol{c}^{\prime}\right) \\
& =(\boldsymbol{D}+\boldsymbol{C})^{-1}\left(-\boldsymbol{f}+\boldsymbol{c}^{\prime}\right)
\end{aligned}
$$

or using (11) to obtain

$$
\begin{aligned}
\boldsymbol{u}^{*} & =(\boldsymbol{D}+\boldsymbol{C})^{-1} \boldsymbol{D} \boldsymbol{u}_{0}^{*} \\
& =\boldsymbol{u}_{0}^{*}+\boldsymbol{M} \boldsymbol{u}_{0}^{*},
\end{aligned}
$$

where $\boldsymbol{C}=\boldsymbol{G}-\boldsymbol{D}$ and $\boldsymbol{M}=-\left[I_{m}+\boldsymbol{C}^{-1} \boldsymbol{D}\right]^{-1}$ with $I_{m}$ denoting an $m \times m$ identity matrix.

When the system is disturbed and has unmodeled dynamics, (13) cannot be practically applied. Accordingly this paper proposes the robust neuro-fuzzy controller that uses the multi-layer fuzzy controller (10) as an alternative of (11) and invokes a decoupling neural network to counteract the interaction by learning as 
follows:

$$
\boldsymbol{u}=\boldsymbol{u}_{0}+\hat{M} \boldsymbol{u}_{0}
$$

where the matrix $\hat{\boldsymbol{M}}$ is chosen as

$$
\hat{\boldsymbol{M}}=-\left(I_{m}+\hat{\boldsymbol{C}}^{-1} \hat{\boldsymbol{D}}\right)^{-1}
$$

and

$$
\hat{\boldsymbol{C}}=\left[\begin{array}{cccc}
0 & \boldsymbol{\omega}_{12}^{\mathrm{T}} \hat{\boldsymbol{g}}_{\omega} & \cdots & \boldsymbol{\omega}_{1 m}^{\mathrm{T}} \hat{\boldsymbol{g}}_{\omega} \\
\boldsymbol{\omega}_{21}^{\mathrm{T}} \hat{\boldsymbol{g}}_{\omega} & 0 & \cdots & \boldsymbol{\omega}_{2 m}^{\mathrm{T}} \hat{\boldsymbol{g}}_{\omega} \\
\vdots & \vdots & \ddots & \vdots \\
\boldsymbol{\omega}_{m 1}^{\mathrm{T}} \hat{\boldsymbol{g}}_{\omega} & \boldsymbol{\omega}_{m 2}^{\mathrm{T}} \hat{\boldsymbol{g}}_{\omega} & \cdots & 0
\end{array}\right]
$$

Fig. 1 illustrates the concept of the robust neuro-fuzzy controller with the decoupling neural network. Using (10), (14), (15) and the matrix inversion lemma [5]

$$
(A+B C D)^{-1}=A^{-1}-A^{-1} B\left(D A^{-1} B+C^{-1}\right)^{-1} D A^{-1}
$$

the robust neuro-fuzzy controller resolves into

$$
\begin{aligned}
\boldsymbol{u} & =\left(I_{m}-\left(I_{m}+\hat{\boldsymbol{C}}^{-1} \hat{\boldsymbol{D}}\right)^{-1}\right) \hat{\boldsymbol{D}}^{-1}\left(-\hat{\boldsymbol{f}}+\boldsymbol{c}^{\prime}-a_{\mathrm{LR}}^{\mathrm{T}} \hat{f}_{\mathrm{LR}}\right) \\
& =\hat{\boldsymbol{G}}^{-1}\left(-\hat{\boldsymbol{f}}+\boldsymbol{c}^{\prime}-a_{\mathrm{LR}}^{\mathrm{T}} \hat{f}_{\mathrm{LR}}\right),
\end{aligned}
$$

where $\hat{\boldsymbol{G}}=\hat{\boldsymbol{C}}+\hat{\boldsymbol{D}}$.

\section{Learning algorithms and performance analysis}

Let $\boldsymbol{\theta}_{i}=\left[\boldsymbol{\theta}_{i}^{\prime \mathrm{T}}, \boldsymbol{\omega}_{i 1}^{\mathrm{T}}, \ldots, \boldsymbol{\omega}_{i m}^{\mathrm{T}}\right]^{\mathrm{T}}$ being bounded by $M_{\theta_{i}}=\left\{\boldsymbol{\theta}_{i}:\left|\boldsymbol{\theta}_{i}\right| \leqslant \boldsymbol{\theta}_{i, \text { Max }}\right\}$, and define the parameters of the best function approximation to be

$$
\begin{aligned}
\boldsymbol{\theta}_{i}^{\prime *} & \equiv \arg \min _{\theta_{i}^{\prime} \in M_{\theta_{i}}}\left[\sup \left|f_{i}-\boldsymbol{\theta}_{i}^{\prime \mathrm{T}} \hat{\boldsymbol{f}}_{\theta}\right|\right], \\
\boldsymbol{\omega}_{i j}^{*} & \equiv \arg \min _{\omega_{i j} \in M_{\theta_{i}}}\left[\sup \left|g_{i j}-\boldsymbol{\omega}_{i j}^{\mathrm{T}} \hat{\boldsymbol{g}}_{\omega}\right|\right] .
\end{aligned}
$$

Applying (18) to (1), then subtracting $\sum_{j=1}^{m} \omega_{i j}^{\mathrm{T}} \hat{\boldsymbol{g}}_{\omega} u_{j}$ and adding $-\boldsymbol{\theta}_{i}^{\prime \mathrm{T}} \hat{\boldsymbol{f}}_{\theta}+c_{i}^{\prime}-a_{\mathrm{LR} i}^{\mathrm{T}} \hat{\mathrm{LR}}_{\mathrm{LR}}$ to the right-hand side, the $i$ th component is obtained as

$$
y_{i}^{\left(r_{i}\right)}=y_{\mathrm{d} i}^{\left(r_{i}\right)}-\boldsymbol{\alpha}_{i}^{\mathrm{T}} e_{i}+\left(\boldsymbol{\theta}_{i}^{\prime * \mathrm{~T}}-\boldsymbol{\theta}_{i}^{\prime \mathrm{T}}\right) \hat{\boldsymbol{f}}_{\theta}+\sum_{j=1}^{m}\left(\boldsymbol{\omega}_{i j}^{* \mathrm{~T}}-\boldsymbol{\omega}_{i j}^{\mathrm{T}}\right) \hat{\boldsymbol{g}}_{\omega} u_{j}+\zeta_{i}-a_{\mathrm{LR} i} \hat{f}_{\mathrm{LR}}
$$

or

$$
\dot{e}_{i}=A_{i} e_{i}-b_{i} \tilde{\boldsymbol{\theta}}_{i}^{\mathrm{T}} \boldsymbol{w}+b_{i}\left(\zeta_{i}-a_{\mathrm{LR} i} \hat{f}_{\mathrm{LR}}\right),
$$


where $\zeta_{i}=\left(f_{i}-\boldsymbol{\theta}_{i}^{* \mathrm{~T}} \hat{\boldsymbol{f}_{\theta}}\right)+\sum_{j=1}^{m}\left(\boldsymbol{g}_{i j}-\boldsymbol{\omega}_{i j}^{* \mathrm{~T}} \hat{\boldsymbol{g}}\right) u_{j}+\boldsymbol{d}_{i}, \tilde{\boldsymbol{\theta}}_{i}=\boldsymbol{\theta}_{i}-\boldsymbol{\theta}_{i}^{*}$ denotes the parameter estimation error, and

$$
A_{i}=\left[\begin{array}{ccccc}
0 & 1 & 0 & \cdots & 0 \\
0 & 0 & 1 & \cdots & 0 \\
\vdots & \vdots & \vdots & \ddots & \vdots \\
0 & 0 & 0 & \cdots & 1 \\
-\alpha_{i 1} & -\alpha_{i 2} & -\alpha_{i 3} & \cdots & -\alpha_{i r_{i}}
\end{array}\right], \quad b_{i}=\left[\begin{array}{c}
0 \\
0 \\
\vdots \\
0 \\
1
\end{array}\right], \quad \boldsymbol{w}=\left[\begin{array}{c}
\hat{\boldsymbol{f}}_{\theta} \\
\hat{\boldsymbol{g}}_{\omega} u_{1} \\
\vdots \\
\hat{\boldsymbol{g}}_{\omega} u_{m}
\end{array}\right]
$$

In the following paragraph, a gradient weight adaptation scheme which shutdown the self-tuning mechanism (i.e. $a_{\mathrm{LR} i}=0$ ) and a robust weight adaptation scheme which applies the self-tuning mechanism (i.e., $a_{\mathrm{LR} i} \neq 0$ ) are proposed and compared.

Gradient weight adaptation scheme: For $a_{\mathrm{LR} i}=0$, the tracking error represented by (21) allows us to use the parameter adaptation law in $[10,13]$

$$
\dot{\boldsymbol{\theta}}_{i}= \begin{cases}0 & \text { if } \boldsymbol{e}^{\mathrm{T}} \boldsymbol{P} \boldsymbol{b} \boldsymbol{b}^{\mathrm{T}} \boldsymbol{P} \boldsymbol{e} \leqslant d_{0}^{2}, \\ \left(I-d_{\theta i} \boldsymbol{\theta}_{i \perp} \boldsymbol{\theta}_{i \perp}^{\mathrm{T}}\right) R_{i}^{-1} b_{i}^{\mathrm{T}} P_{i} e_{i} \boldsymbol{w} & \text { otherwise }\end{cases}
$$

with

$$
d_{\theta i}= \begin{cases}0 & \text { if } \boldsymbol{\theta}_{i \perp}^{\mathrm{T}}\left(R_{i}^{-1} b_{i}^{\mathrm{T}} P_{i} e_{i} \boldsymbol{w}\right) \leqslant 0 \\ \min \left[1, \operatorname{dist}\left(\boldsymbol{\theta}_{i}, M_{\theta_{i}}\right) / \varepsilon_{\theta}\right] & \text { otherwise }\end{cases}
$$

where $R_{i}$ is a diagonal matrix with positive diagonal elements, $\boldsymbol{b}=$ Block $\operatorname{diag}\left[b_{1}, \ldots, b_{m}\right], \quad \boldsymbol{P}=$ Block diag $\left[P_{1}, \ldots, P_{m}\right]$, and $P_{i}=P_{i}^{\mathrm{T}}$ is the solution of the following Riccati-like equation:

$$
P_{i} A_{i}+A_{i}^{\mathrm{T}} P_{i}+\frac{1}{\varrho^{2}} P_{i} b_{i} b_{i}^{\mathrm{T}} P_{i}+Q_{i}=0
$$

with the design parameters $Q_{i}>0$ and $\rho>0, M_{\theta i}^{\varepsilon}$ denotes the union of $M_{\theta i}$ and its boundary layer with thickness $\varepsilon_{\theta}$, the prefix $\partial$ denotes the boundary, and $\boldsymbol{\theta}_{i \perp}=\boldsymbol{\theta}_{i} /\left|\boldsymbol{\theta}_{i}\right|$ is the unit normal vector. The adaptation is turned-off when the tracking error is smaller than some threshold. The deadzone is to stop updating the parameters when the excitation is insufficient to distinguish between the regression signal and the noise. By referring to the result in [10], this adaptive law has the following property:

Theorem 1. Let $\zeta=\left[\zeta_{1}, \ldots, \zeta_{m}\right]^{\mathrm{T}}, Q=$ Block diag $\left[Q_{1}, \ldots, Q_{m}\right]$ and assume that there exists $\bar{\zeta}=$ Sup $_{\boldsymbol{x}, t}\left\|\zeta_{i}\right\|^{2}$, and $\left\|e_{i}\right\|<\delta_{1},\left\|\tilde{\boldsymbol{\theta}_{i}}\right\|<\delta_{2}, \delta_{1}$ and $\delta_{2}$ are small enough, the adaptive law (23) guarantees

(1) $\boldsymbol{\theta}_{i}$ and the control input $\boldsymbol{u}$ are bounded.

(2) $\boldsymbol{e}$ converges to the residual set $\left\{\boldsymbol{e}: \boldsymbol{e}^{\mathrm{T}} Q \boldsymbol{e} \leqslant \rho^{2 \bar{\zeta}}\right.$ or $\left.\boldsymbol{e}^{\mathrm{T}} \boldsymbol{P} \boldsymbol{b} \boldsymbol{b}^{\mathrm{T}} \boldsymbol{P} \boldsymbol{e} \leqslant d_{0}^{2}\right\}$. Moreover, in the special case that $\|\zeta\| \leqslant\left(1 / 2 \rho^{2}\right) d_{0}$, e converges to the dead-zone $\left\{\boldsymbol{e}: \boldsymbol{e}^{\mathrm{T}} \boldsymbol{P} \boldsymbol{b} \boldsymbol{b}^{\mathrm{T}} \boldsymbol{P} \boldsymbol{e} \leqslant d_{0}^{2}\right\}$.

Robust weight adaptation scheme: To counteract the equivalent uncertainty, the self-tuning mechanism $a_{\mathrm{LR} i} \hat{f}_{\mathrm{LR}}$ is employed. The parameter $a_{\mathrm{LR} i}$ is chosen as $a_{\mathrm{LR} i}\left(\vartheta_{i}\right)=\vartheta_{i} \tanh \left(b_{i}^{\mathrm{T}} P_{i} e_{i} \hat{f}_{\mathrm{LR}} / \varepsilon\right)$ where $\vartheta_{i}$ is an auxiliary adjustable parameter and $\varepsilon$ is a small positive constant. Using the following assumption:

Assumption 1. There exists the smallest non-negative parameter values $\vartheta_{i}^{*} \geqslant 0$ such that for all $\boldsymbol{x} \in R^{n}$ and $t \in R_{+}$

$$
\left|\zeta_{i}\right| \leqslant \vartheta_{i}^{*} \hat{f}_{\mathrm{LR}}
$$


And let $M_{\vartheta_{i}}=\left\{\vartheta_{i}:\left|\vartheta_{i}\right|<\vartheta_{i, \max }\right\}$ be the bound of $\vartheta_{i}, M_{\vartheta_{i}}^{\varepsilon}$ be the union of $M_{\vartheta_{i}}$ and its boundary layer of thickness $\varepsilon_{\vartheta}$. We propose the following smooth robust weight adaptation scheme:

$$
\dot{\boldsymbol{\theta}}_{i}= \begin{cases}0 & \text { if } \boldsymbol{e}^{\mathrm{T}} \boldsymbol{P} \boldsymbol{b} \boldsymbol{b}^{\mathrm{T}} \boldsymbol{P} \boldsymbol{e} \leqslant d_{0}^{2}, \\ \left(I-d_{\theta_{i}} \boldsymbol{\theta}_{i \perp} \boldsymbol{\theta}_{i \perp}^{\mathrm{T}}\right) R_{i}^{-1}\left[\boldsymbol{w} b_{i}^{\mathrm{T}} P_{i} e_{i}-\sigma_{1}\left(\boldsymbol{\theta}_{i}-\boldsymbol{\theta}_{i 0}\right)\right] & \text { otherwise }\end{cases}
$$

with

$$
d_{\theta_{i}}= \begin{cases}0 & \text { if } \boldsymbol{\theta}_{i \perp}^{\mathrm{T}}\left[\boldsymbol{w} b_{i}^{\mathrm{T}} P_{i} e_{i}-\sigma_{1}\left(\boldsymbol{\theta}_{i}-\boldsymbol{\theta}_{i 0}\right)\right] \leqslant 0, \\ \min \left[1, \operatorname{dist}\left(\boldsymbol{\theta}_{i}, M_{\theta_{i}}\right) / \varepsilon_{\theta}\right] & \text { otherwise }\end{cases}
$$

and

$$
\dot{\vartheta}_{i}= \begin{cases}0 & \text { if } \boldsymbol{e}^{\mathrm{T}} \boldsymbol{P} \boldsymbol{b} \boldsymbol{b}^{\mathrm{T}} \boldsymbol{P} \boldsymbol{e} \leqslant d_{0}^{2}, \\ \left(1-d_{\vartheta_{i}}\right) r_{\vartheta_{i}}^{-1}\left[w_{i}^{\prime} b_{i}^{\mathrm{T}} P_{i} e_{i}-\sigma_{2}\left(\vartheta_{i}-\vartheta_{i 0}\right)\right] & \text { otherwise }\end{cases}
$$

with

$$
\begin{aligned}
& d_{\vartheta_{i}}= \begin{cases}0 & \text { if } \vartheta_{i}\left[w_{i}^{\prime} b_{i}^{\mathrm{T}} P_{i} e_{i}-\sigma_{2}\left(\vartheta_{i}-\vartheta_{i 0}\right)\right] \leqslant 0, \\
\min \left[1, \operatorname{dist}\left(\vartheta_{i}, M_{\vartheta_{i}}\right) / \varepsilon_{\vartheta}\right] & \text { otherwise. }\end{cases} \\
& w_{i}^{\prime}=\hat{f}_{\mathrm{LR}} \tanh \left(\frac{b_{i}^{\mathrm{T}} P_{i} e_{i} \hat{f}_{\mathrm{LR}}}{\varepsilon}\right),
\end{aligned}
$$

where $R_{i}$ is a diagonal matrix with positive diagonal elements, $P_{i}$ is a symmetric positive-definite matrix satisfying the Lyapunov equation $A_{i}^{\mathrm{T}} P_{i}+P_{i} A_{i}=-Q_{i}^{\prime}$, with the design parameters $Q_{i}^{\prime}>0$, and $\sigma_{1}$ and $\sigma_{2}$ are chosen small but positive constant to keep $\boldsymbol{\theta}_{i}$ and $\vartheta_{i}$ from growing unbounded.

Theorem 2. Consider the nonlinear composite system (1) with controller (18), the parameter adaptation schemes (27) and (29) operating in the bounded state $\boldsymbol{x} \in \Omega$. Then

(1) $\boldsymbol{\theta}_{i}, \vartheta_{i}$ and the control input $\boldsymbol{u}$ are uniformly ultimately bounded.

(2) Given any $\rho$ satisfying $\rho^{*}<\rho$ where

$$
\rho^{*}=\frac{\sum_{i=1}^{m}\left[\sigma_{1}\left(\boldsymbol{\theta}_{i}^{*}-\boldsymbol{\theta}_{i 0}\right)^{\mathrm{T}}\left(\boldsymbol{\theta}_{i}^{*}-\boldsymbol{\theta}_{i 0}\right)+\sigma_{2}\left(\vartheta_{i}^{*}-\vartheta_{i 0}\right)^{2}+2 \kappa \vartheta_{i}^{M} \varepsilon\right]}{\min _{i} \min \left\{\lambda_{\min }\left(Q_{i}^{\prime}\right) / \lambda_{\max }\left(P_{i}\right), \sigma_{1} / \lambda_{\max }\left(R_{i}\right), \sigma_{2} / \gamma_{\vartheta}\right\}}
$$

with $\vartheta_{i}^{M} \equiv \max \left\{\vartheta_{i}^{*}, \vartheta_{i 0}\right\}$ and $\kappa$ being a constant that satisfies $\kappa=\mathrm{e}^{-(\kappa+1)}$, i.e., $\kappa=0.2785$, there exists $T$ such that for $T \leqslant t \leqslant \infty$ the tracking error e converges to the residual set

$$
\left\{\boldsymbol{e}: \boldsymbol{e}^{\mathrm{T}} \boldsymbol{P} \boldsymbol{e} \leqslant \rho \text { or } \boldsymbol{e}^{\mathrm{T}} \boldsymbol{P} \boldsymbol{b} \boldsymbol{b}^{\mathrm{T}} \boldsymbol{P} \boldsymbol{e} \leqslant d_{0}^{2}\right\} .
$$

Proof. Let $V_{\theta}$ and $V_{\vartheta}$ be positive-definite functions of the forms $V_{\theta}=\frac{1}{2} \sum_{i=1}^{m}\left(\boldsymbol{\theta}_{i}^{\mathrm{T}} \boldsymbol{\theta}_{i}\right), V_{\vartheta}=\frac{1}{2} \sum_{i=1}^{m} \vartheta^{2}$. Their time derivative are $\dot{V}_{\theta}=\sum_{i=1}^{m} \boldsymbol{\theta}_{i}^{\mathrm{T}} \dot{\theta}_{i}$ and $\dot{V}_{\vartheta}=\sum_{i=1}^{m} \vartheta_{i}^{\mathrm{T}} \dot{\vartheta}_{i}$, respectively. If the first line of (28) is true then $d_{\theta_{i}}=0$, and the conclusion $\dot{V}_{\theta} \leqslant 0$ is trivial. If the second line of (28) is true then $d_{\theta_{i}}<1$ and $\boldsymbol{\theta}_{i} \in M_{\theta_{i}}^{\varepsilon}$ (but $\boldsymbol{\theta}_{i} \notin \partial M_{\theta_{i}}^{\varepsilon}$ ). Therefore, either $\dot{V}_{\theta} \leqslant 0$ or $\boldsymbol{\theta}_{i} \in M_{\theta_{i}}^{\varepsilon}$ is obtained. Similarly we have either $\dot{V}_{\vartheta}<0$ or $\vartheta_{i} \in M_{\vartheta_{i}}^{\varepsilon}$. Therefore, the boundedness of $\boldsymbol{\theta}_{i}, \vartheta_{i}$, and $\boldsymbol{u}$ is guaranteed. To show the performance of the closed-loop system formed 
by (1), (18), (27), and (29), we choose the following positive-definite functions:

$$
\boldsymbol{V}=V_{1}+\cdots+V_{m},
$$

where

$$
V_{i}= \begin{cases}\frac{1}{2} d_{0}^{2}+\frac{1}{2} \tilde{\boldsymbol{\theta}}_{i}^{\mathrm{T}} \boldsymbol{R}_{i} \tilde{\boldsymbol{\theta}}_{i}+\frac{1}{2} r_{\vartheta_{i}} \tilde{\vartheta}_{i}^{2} & \text { if } \boldsymbol{e}^{\mathrm{T}} \boldsymbol{P} \boldsymbol{b} \boldsymbol{b}^{\mathrm{T}} \boldsymbol{P} \boldsymbol{e} \leqslant d_{0}^{2}, \\ \frac{1}{2} e_{i}^{\mathrm{T}} P_{i} e_{i}+\frac{1}{2} \tilde{\boldsymbol{\theta}}_{i}^{\mathrm{T}} \boldsymbol{R}_{i} \tilde{\boldsymbol{\theta}}_{i}+\frac{1}{2} r_{\vartheta_{i}} \tilde{\vartheta}_{i}^{2} & \text { otherwise }\end{cases}
$$

$\tilde{\vartheta}_{i}=\vartheta_{i}-\vartheta_{i}^{M}$ are the auxiliary adjustable parameter error and $\vartheta_{i}^{M} \equiv \max \left\{\vartheta_{i}^{*}, \vartheta_{i 0}\right\}$. Taking the derivative of $V_{i}$ along the trajectories of the closed-loop system and taking (21), (27), and (29) into account we obtain: $\dot{V}_{i}=0$ for $\boldsymbol{e}^{\mathrm{T}} \boldsymbol{P} \boldsymbol{b} \boldsymbol{b}^{\mathrm{T}} \boldsymbol{P} \boldsymbol{e} \leqslant d_{0}^{2}$, and

$$
\begin{aligned}
\dot{V}_{i}= & e_{i}^{\mathrm{T}} P_{i}\left(A_{i} e_{i}-b_{i} \tilde{\boldsymbol{\theta}}_{i}^{\mathrm{T}} \boldsymbol{w}+b_{i}\left(\zeta_{i}-a_{\mathrm{LR} i} \hat{f}_{\mathrm{LR}}\right)\right)+\tilde{\boldsymbol{\theta}}_{i}^{\mathrm{T}}\left(I-d_{\theta} \boldsymbol{\theta}_{i \perp} \boldsymbol{\theta}_{i \perp}^{\mathrm{T}}\right)\left[\boldsymbol{w} b_{i}^{\mathrm{T}} P_{i} e_{i}\right. \\
& \left.-\sigma_{1}\left(\boldsymbol{\theta}_{i}-\boldsymbol{\theta}_{i 0}\right)\right]+\tilde{\vartheta}_{i}\left(1-d_{\vartheta}\right)\left[w_{i}^{\prime} b_{i}^{\mathrm{T}} P_{i} e_{i}-\sigma_{2}\left(\vartheta_{i}-\vartheta_{i 0}\right)\right] \\
= & \frac{1}{2} e_{i}^{\mathrm{T}}\left(A_{i}^{\mathrm{T}} P_{i}+P_{i} A_{i}\right) e_{i}-e_{i}^{\mathrm{T}} P_{i} b_{i} \tilde{\boldsymbol{\theta}}_{i}^{\mathrm{T}} \boldsymbol{w}+e_{i}^{\mathrm{T}} P_{i} b_{i}\left(\zeta_{i}-\vartheta_{i} w_{i}^{\prime}\right) \\
& +\tilde{\boldsymbol{\theta}}_{i}^{\mathrm{T}} \boldsymbol{w} b_{i}^{\mathrm{T}} P_{i} e_{i}-\sigma_{1} \tilde{\boldsymbol{\theta}}_{i}^{\mathrm{T}}\left(\boldsymbol{\theta}_{i}-\boldsymbol{\theta}_{i 0}\right)+\tilde{\vartheta}_{i} w_{i}^{\prime} b_{i}^{\mathrm{T}} P_{i} e_{i}-\sigma_{2} \tilde{\vartheta}_{i}\left(\vartheta_{i}-\vartheta_{i 0}\right) \\
& -d_{\theta} \tilde{\boldsymbol{\theta}}_{i}^{\mathrm{T}} \boldsymbol{\theta}_{i \perp} \boldsymbol{\theta}_{i \perp}^{\mathrm{T}}\left[\boldsymbol{w} b_{i}^{\mathrm{T}} P_{i} e_{i}-\sigma_{1}\left(\boldsymbol{\theta}_{i}-\boldsymbol{\theta}_{i 0}\right)\right]-d_{\vartheta} \tilde{\vartheta}_{i}\left[w_{i}^{\prime} b_{i}^{\mathrm{T}} P_{i} e_{i}-\sigma_{2}\left(\vartheta_{i}-\vartheta_{i 0}\right)\right]
\end{aligned}
$$

for $\boldsymbol{e}^{\mathrm{T}} \boldsymbol{P} \boldsymbol{b} \boldsymbol{b}^{\mathrm{T}} \boldsymbol{P} \boldsymbol{e}>d_{0}^{2}$. By (28), if $\boldsymbol{\theta}_{i \perp}^{\mathrm{T}}\left[\boldsymbol{w}_{i} b_{i}^{\mathrm{T}} P_{i} e_{i}-\sigma_{1}\left(\boldsymbol{\theta}_{i}-\boldsymbol{\theta}_{i 0}\right)\right] \leqslant 0$, we have $d_{\theta_{i}}=0$ and the last term of the above equation is equal to zero. When $\boldsymbol{\theta}_{i \perp}^{\mathrm{T}}\left[\boldsymbol{w}_{i} b_{i}^{\mathrm{T}} P_{i} e_{i}-\sigma_{1}\left(\boldsymbol{\theta}_{i}-\boldsymbol{\theta}_{i 0}\right)\right]>0$, if $\boldsymbol{\theta}_{i} \in M_{\theta_{i}}$ we also have $d_{\theta_{i}}=0$ and the above conclusion holds. If $\boldsymbol{\theta}_{i} \notin M_{\theta_{i}}$ and suppose that $M_{\theta_{i}}$ and $M_{\vartheta_{i}}$ are appropriately selected such that $\boldsymbol{\theta}_{i}^{*}$ and $\vartheta_{i}^{*}$ are in the interior of $M_{\theta_{i}}$ and $M_{\vartheta_{i}}$, respectively, we obtain

$$
\begin{aligned}
\tilde{\boldsymbol{\theta}}_{i}^{\mathrm{T}} \boldsymbol{\theta}_{i \perp} & =\left(\boldsymbol{\theta}_{i}-\boldsymbol{\theta}_{i}^{*}\right)^{\mathrm{T}} \boldsymbol{\theta}_{i} /\left|\boldsymbol{\theta}_{i}\right| \\
& =\frac{1}{2}\left[\left(\boldsymbol{\theta}_{i}-\boldsymbol{\theta}_{i}^{*}\right)^{\mathrm{T}}\left(\boldsymbol{\theta}_{i}-\boldsymbol{\theta}_{i}^{*}\right)+\boldsymbol{\theta}_{i}^{\mathrm{T}} \boldsymbol{\theta}_{i}-\boldsymbol{\theta}_{i}^{* \mathrm{~T}} \boldsymbol{\theta}_{i}^{* 2}\right] /\left|\boldsymbol{\theta}_{i}\right| \\
& \geqslant 0
\end{aligned}
$$

or

$$
\tilde{\boldsymbol{\theta}}_{i}^{\mathrm{T}} \boldsymbol{\theta}_{i \perp} \boldsymbol{\theta}_{i \perp}^{\mathrm{T}}\left[\boldsymbol{w} b_{i}^{\mathrm{T}} P_{i} e_{i}-\sigma_{1}\left(\boldsymbol{\theta}_{i}-\boldsymbol{\theta}_{i 0}\right)\right] \geqslant 0 .
$$

In a similar way, it can be shown that

$$
\tilde{\vartheta}_{i}\left[w_{i}^{\prime} b_{i}^{\mathrm{T}} P_{i} e_{i}-\sigma_{2}\left(\vartheta_{i}-\vartheta_{i 0}\right)\right] \geqslant 0 \text {. }
$$

Therefore,

$$
\begin{aligned}
\dot{V}_{i} \leqslant & \frac{1}{2} e_{i}^{\mathrm{T}}\left(A_{i}^{\mathrm{T}} P_{i}+P_{i} A_{i}\right) e_{i}+e_{i}^{\mathrm{T}} P_{i} b_{i}\left(\zeta_{i}-\vartheta^{M} w_{i}^{\prime}\right)-\sigma_{1} \tilde{\boldsymbol{\theta}}_{i}^{\mathrm{T}}\left(\boldsymbol{\theta}_{i}-\boldsymbol{\theta}_{i 0}\right) \\
& -\sigma_{2} \tilde{\vartheta}_{i}\left(\vartheta_{i}-\vartheta_{i 0}\right) .
\end{aligned}
$$

Using Assumption 1, the second term on the right-hand side satisfies the inequality

$$
\begin{aligned}
e_{i}^{\mathrm{T}} P_{i} b_{i}\left(\zeta_{i}-\vartheta^{M} w_{i}^{\prime}\right) & \leqslant\left|e_{i}^{\mathrm{T}} P_{i} b_{i}\right| \vartheta_{i}^{*} \hat{f}_{\mathrm{LR}}-e_{i}^{\mathrm{T}} P_{i} b_{i} \vartheta^{M} w_{i}^{\prime} \\
& \leqslant \vartheta_{i}^{M}\left(\left|e_{i}^{\mathrm{T}} P_{i} b_{i}\right| \hat{f}_{\mathrm{LR}}-e_{i}^{\mathrm{T}} P_{i} b_{i} w_{i}^{\prime}\right)
\end{aligned}
$$




$$
\begin{aligned}
& =\vartheta_{i}^{M}\left(\left|e_{i}^{\mathrm{T}} P_{i} b_{i} \hat{f}_{\mathrm{LR}}\right|-e_{i}^{\mathrm{T}} P_{i} b_{i} \hat{f}_{\mathrm{LR}} \tanh \left(\frac{e_{i}^{\mathrm{T}} P_{i} b_{i} \hat{f}_{\mathrm{LR}}}{\varepsilon}\right)\right) \\
& \leqslant \vartheta_{i}^{M} \kappa \varepsilon .
\end{aligned}
$$

Since the following fact can be shown easily by straightforward algebraic manipulation.

\section{Claim 1.}

$$
0 \leqslant|r|-r \tanh \left(\frac{r}{\varepsilon}\right) \leqslant \kappa \varepsilon
$$

for any $\gamma \in R$. Furthermore, it can be readily shown that

$$
\begin{aligned}
& \tilde{\boldsymbol{\theta}}_{i}^{\mathrm{T}}\left(\boldsymbol{\theta}_{i}-\boldsymbol{\theta}_{i 0}\right)=\frac{1}{2} \tilde{\boldsymbol{\theta}}_{i}^{\mathrm{T}} \tilde{\boldsymbol{\theta}}_{i}+\frac{1}{2}\left(\boldsymbol{\theta}_{i}-\boldsymbol{\theta}_{i 0}\right)^{\mathrm{T}}\left(\boldsymbol{\theta}_{i}-\boldsymbol{\theta}_{i 0}\right)-\frac{1}{2}\left(\boldsymbol{\theta}_{i}^{*}-\boldsymbol{\theta}_{i 0}\right)^{\mathrm{T}}\left(\boldsymbol{\theta}_{i}^{*}-\boldsymbol{\theta}_{i 0}\right), \\
& \tilde{\vartheta}_{i}\left(\vartheta_{i}-\vartheta_{i 0}\right)=\frac{1}{2} \tilde{\vartheta}_{i}^{2}+\frac{1}{2}\left(\vartheta_{i}-\vartheta_{i 0}\right)^{2}-\frac{1}{2}\left(\vartheta_{i}^{*}-\vartheta_{i 0}\right)^{2} .
\end{aligned}
$$

Therefore,

$$
\begin{aligned}
\dot{V}_{i} & \leqslant-\frac{1}{2} e_{i}^{\mathrm{T}}\left(Q_{i}^{\prime}\right) e_{i}-\frac{\sigma_{1}}{2} \tilde{\boldsymbol{\theta}}_{i}^{\mathrm{T}} \tilde{\boldsymbol{\theta}}_{i}-\frac{\sigma_{2}}{2} \tilde{\vartheta}_{i}^{2}+\frac{\sigma_{1}}{2}\left(\boldsymbol{\theta}_{i}^{*}-\boldsymbol{\theta}_{i 0}\right)^{\mathrm{T}}\left(\boldsymbol{\theta}_{i}^{*}-\boldsymbol{\theta}_{i 0}\right)+\frac{\sigma_{2}}{2}\left(\vartheta_{i}^{*}-\vartheta_{i 0}\right)^{2}+\kappa \vartheta_{i}^{M} \varepsilon \\
& \leqslant-a_{i} V_{i}+\lambda_{i},
\end{aligned}
$$

where

$$
a_{i} \equiv \min \left\{\frac{\lambda_{\min }\left(Q_{i}^{\prime}\right)}{\lambda_{\max }\left(P_{i}\right)}, \frac{\sigma_{1}}{\lambda_{\max }\left(R_{i}\right)}, \frac{\sigma_{2}}{\gamma_{\vartheta_{i}}}\right\}
$$

and

$$
\lambda_{i}=\frac{\sigma_{1}}{2}\left(\boldsymbol{\theta}_{i}^{*}-\boldsymbol{\theta}_{i 0}\right)^{\mathrm{T}}\left(\boldsymbol{\theta}_{i}^{*}-\boldsymbol{\theta}_{i 0}\right)+\frac{\sigma_{2}}{2}\left(\vartheta_{i}^{\prime}-\vartheta_{i 0}\right)^{2}+\vartheta_{i}^{M} \kappa \varepsilon
$$

or

$$
\dot{\boldsymbol{V}} \leqslant-a \boldsymbol{V}+\lambda
$$

where $a=\min _{i} a_{i}$ and $\lambda=\sum_{i=1}^{m} \lambda_{i}$. The differential inequality (45) satisfies

$$
0 \leqslant \boldsymbol{V}(t) \leqslant \frac{\lambda}{a}+\left(\boldsymbol{V}(0)-\frac{\lambda}{a}\right) \mathrm{e}^{-a t} .
$$

Therefore $e_{i}, \boldsymbol{\theta}_{i}, \vartheta_{i}$ are uniformly ultimately bounded. Let $\rho^{*}=2 \lambda / a$ then from (46) we readily obtain (33).

Remark 1. The gradient weight adaptation scheme in [10] requires the assumption that the tracking error and weight errors are initially bounded and sufficiently small. In our construction of the robust weight adaptation scheme by using the self-tuning mechanism, $a_{\mathrm{LR} i} \hat{f}_{\mathrm{LR}}$, the assumption of small initial weight errors is released.

Remark 2. From (32), if the design constants $\varepsilon, \sigma_{1}, \sigma_{2}, \gamma_{\vartheta}, Q_{i}, P_{i}$ and $R_{i}$ are appropriately chosen, whether $\boldsymbol{\theta}_{i 0}$ and $\vartheta_{i 0}$ are close to $\boldsymbol{\theta}_{i}^{*}$ and $\vartheta_{i}^{*}$ or not, it is possible to make $\rho^{*}$ as small as desired and therefore better tracking performance can be achieved. 


\section{Performance comparisons}

\subsection{Simulation setup}

A two-link robot is simulated to compare the robustness of the gradient weight adaptation scheme and the proposed robust weight adaptation scheme.

The equations of motion of the arm can be expressed in the matrix form as follows:

$$
\begin{aligned}
& {\left[\begin{array}{c}
\left(m_{1}+m_{2}\right) r_{1}^{2}+m_{2} r_{2}^{2}+2 m_{2} r_{1} r_{2} c_{2}+J_{1} \\
m_{2} r_{2}^{2}+m_{2} r_{1} r_{2} c_{2}+m_{2} r_{1} r_{2} c_{2} \\
m_{2} r_{2}^{2}+J_{2}
\end{array}\right] \quad\left[\begin{array}{l}
\ddot{q}_{1} \\
\ddot{q}_{2}
\end{array}\right]} \\
& \quad+\left[\begin{array}{c}
-m_{2} r_{1} r_{2} s_{2} \dot{q}_{1}\left(\dot{q}_{1}+\dot{q}_{2}\right) \\
m_{2} r_{1} r_{2} s_{2} \dot{q}_{2}^{2}
\end{array}\right]+\left[\begin{array}{c}
\left(\left(m_{1}+m_{2}\right) l_{1} c_{2}+m_{2} l_{2} c_{12}\right) g \\
\left(m_{2} l_{2} c_{12}\right) g
\end{array}\right]=\left[\begin{array}{l}
u_{1} \\
u_{2}
\end{array}\right]+\left[\begin{array}{l}
d_{1} \\
d_{2}
\end{array}\right],
\end{aligned}
$$

where $m_{1}, m_{2}, J_{1}, J_{2}, r_{1}=0.5 l_{1}$, and $r_{2}=0.5 l_{2}$ are the mass, the moment of inertia, the half length of link 1 and 2 , and $c_{1} \equiv \cos \left(q_{1}\right), s_{12} \equiv \sin \left(q_{1}+q_{2}\right)$, etc. The combined effects of friction and the external torque disturbance are

$$
\begin{aligned}
& d_{1}=2.0 \sin \left(\dot{q}_{1}\right)+2.5 \sin \left(\dot{q}_{2}\right)+0.5 \sin (t), \\
& d_{2}=5.0 \sin \left(\dot{q}_{1}\right)+4.0 \sin \left(\dot{q}_{2}\right)+0.4 \sin (t) .
\end{aligned}
$$

In the control experiments described below, the kinematics and inertial parameters of the arm are chosen as $l_{1}=2.04 \mathrm{~m}, l_{2}=1.66 \mathrm{~m}, J_{1}=J_{2}=4.5 \mathrm{~kg} \mathrm{~m}, m_{1}=0.60 \mathrm{~kg}, m_{2}=7.02 \mathrm{~kg}$, respectively. The excessive ratio between $m_{1}$ and $m_{2}$ is to emphasize the load effect. The robot is given the following target joint rotations:

$$
\begin{aligned}
& q_{1 d}=\pi / 12 \sin (0.5 \pi t) \\
& q_{2 d}=2.5 \pi / 12 \cos (0.5 \pi t)+2.5 \pi / 24 \cos (0.5 \pi t)
\end{aligned}
$$

with the initial states $q_{1}(0)=1.5 \mathrm{rad}, q_{2}(0)=-1.2 \mathrm{rad}, \dot{q}_{1}(0)=0 \mathrm{rad} / \mathrm{s}, \dot{q}_{2}(0)=0 \mathrm{rad} / \mathrm{s}$.

In (27) and (29), the design parameter are given by $Q_{1}=Q_{2}=10 I_{2 \times 2}, \quad \boldsymbol{R}_{1}=$ Block diag $\left[0.01 I_{81 \times 81}\right.$, $\left.32000 I_{81 \times 81}, \quad 20000 I_{81 \times 81}\right], \quad \boldsymbol{R}_{2}=$ Block diag $\left[0.025 I_{81 \times 81}, \quad 20000 I_{81 \times 81}, \quad 3200 I_{81 \times 81}\right], \quad \rho=0.01, \quad r_{\vartheta_{1}}=r_{\vartheta_{2}}=$ $0.025, \sigma_{1}=\sigma_{2}=0.002$, and $\varepsilon=0.005$. The membership functions of state $q_{1}, \dot{q}_{1}, q_{2}$, and $\dot{q}_{2}$ (represented by generic variable $x_{k}$ ) for $M=3^{4}=81$ regular rule partitions are defined as $\{\mathrm{NB}, \mathrm{ZE}, \mathrm{PB}\}$ where NB: $A_{k}^{j}\left(x_{k}\right)=$ $\exp \left(-4\left(x_{k}+1.8\right)^{2}\right), \mathrm{ZE}: A_{k}^{j}\left(x_{k}\right)=\exp \left(-4 x_{k}^{2}\right)$, and PB: $A_{k}^{j}\left(x_{k}\right)=\exp \left(-4\left(x_{k}-1.8\right)^{2}\right)$.

For the purpose of comparison, computer simulations are also carried out using the proposed controller with and without partial knowledge about the robot, i.e., rough mathematical model (but unknown disturbances) and nominal parameters $l_{1}^{0}=2.0 \mathrm{~m}, l_{2}^{0}=1.6 \mathrm{~m}, J_{1}^{0}=4.8 \mathrm{~kg}, J_{2}^{0}=5.1 \mathrm{~kg} \mathrm{~m}, m_{1}^{0}=0.48 \mathrm{~kg}, m_{2}^{0}=6.30 \mathrm{~kg}$.

In the case that the nominal robot parameters are known a priori, through the training data $\left\{\boldsymbol{x}^{(k)}\right\}$, the initial parameters $\boldsymbol{\theta}_{i}^{\prime}$ and $\omega_{i j}$ are chosen based on the element-by-element minimization of the following objective function:

$$
\begin{aligned}
& \sum_{k} \mid f_{i}^{0}\left(\boldsymbol{x}^{(k)} \mid \text { nominal robot parameters }\right)-\left.\boldsymbol{\theta}_{i}^{\prime \mathrm{T}} \hat{\boldsymbol{f}}_{\theta}\right|^{2}, \\
& \sum_{k} \mid g_{i j}^{0}\left(\boldsymbol{x}^{(k)} \mid \text { nominal robot parameters }\right)-\left.\boldsymbol{\omega}_{i j}^{\mathrm{T}} \hat{\boldsymbol{g}}_{\omega}\right|^{2} .
\end{aligned}
$$

We choose 32 testing points either the sampled points along the desired trajectories or points near them, for the training data $\boldsymbol{x}^{(k)}$. If there are no nominal robot parameters, the elements in $\boldsymbol{\theta}_{i}^{\prime}$ and $\boldsymbol{\omega}_{i j}$ are chosen randomly in the interval $(-10,10)$ and $(-2,2)$, respectively. 


\subsection{Results and discussion}

Figs. 3 and 4 show the tracking performance using (a) the gradient weight adaptive scheme and (b) the robust weight adaptive scheme with and without nominal robot parameters. The solid and dashed lines correspond to the desired and controlled robot-arm angle trajectories, respectively. In comparing the set of response (a) with (b) in Figs. 3 and 4, one can conclude that the tracking behavior is much better in the robust weight adaptive scheme case. While the gradient weight adaptive scheme reaches a large error residual set, the error in the robust weight adaptive scheme continues to decay around zero. This is exactly the difference between these two schemes. The gradient weight adaptive scheme guarantees that the quadratic error $\boldsymbol{e}^{\mathrm{T}} Q \boldsymbol{e}$ will be smaller than a certain bound, $\rho^{2} \bar{\zeta}$, however, $\bar{\zeta}$ varies with the control effort $\boldsymbol{u}$ and the disturbance $\boldsymbol{d}$. To reduce the error, one has to reduce the design parameter $\rho$ which will cause larger input effort and then yield larger $\bar{\zeta}$. It seems that there is some limitation on reducing the error residual set. Since the error residual set $\rho^{*}$ does not directly depend on the magnitude of control effort (see Eq. (33)), the robust adaptive scheme requires the design constants $\varepsilon, \sigma_{1}, \sigma_{2}, r_{\vartheta}, Q_{i}^{\prime}, P_{i}$ and $R_{i}$ to be just appropriately chosen such that $\rho^{*}$ is reduced. This is the reason why a smaller tracking error residual set can be achieved. On the other hand, in comparing Fig. 3(a) with Fig. 4(a), it is clear and expected that the tracking performance is much better in the case that $\boldsymbol{\theta}_{i}^{\prime}$ and $\boldsymbol{\omega}_{i j}$ are selected in advance according to nominal robot parameters. Nevertheless, as shown in Fig. 3(b) and Fig. 4(b) significant improvements of system tracking performance are achieved after applying the robust weight adaptation scheme even without a priori knowledge of the nominal parameters of the robot.

\section{Conclusion}

A novel robust neuro-fuzzy controller has been developed successfully for the tracking control of composite multivariable systems with uncertainties. The controller uses a decoupling neural network to counteract the interaction among the interconnected subsystems. The system robustness against the effect of equivalent uncertainties for unknown plant dynamics and disturbance is mainly obtained by self-tuning the consequent membership functions of the fuzzy part. A robust weight adaptation scheme with self-tuning on the consequent membership functions has been derived and shown to be able to considerably reduce the tracking error residual set by automatically adapting the controller to react to the uncertainties. The overall system has been proved to be uniform ultimate bounded. And the analysis gives an account of the relationship between control performance and the design parameters of the neuro-fuzzy controller, which was obscure previously in the theory of fuzzy/neural control. Comparison of the proposed method with an adaptive neuro-fuzzy control system without self-tuning the consequent membership function has been carried out via theoretical analysis and simulations. The results show that the proposed method is superior in both tracking performance and robustness. The effectiveness of this design has been investigated and demonstrated by an example of robot control.

\section{Acknowledgements}

The authors would like to thank the anonymous referees for their valuable suggestions and comments. The financial support for this research from the National Science Council of Taiwan, R.O.C. under NSC 88-2212E-002-070 is gratefully acknowledged. 

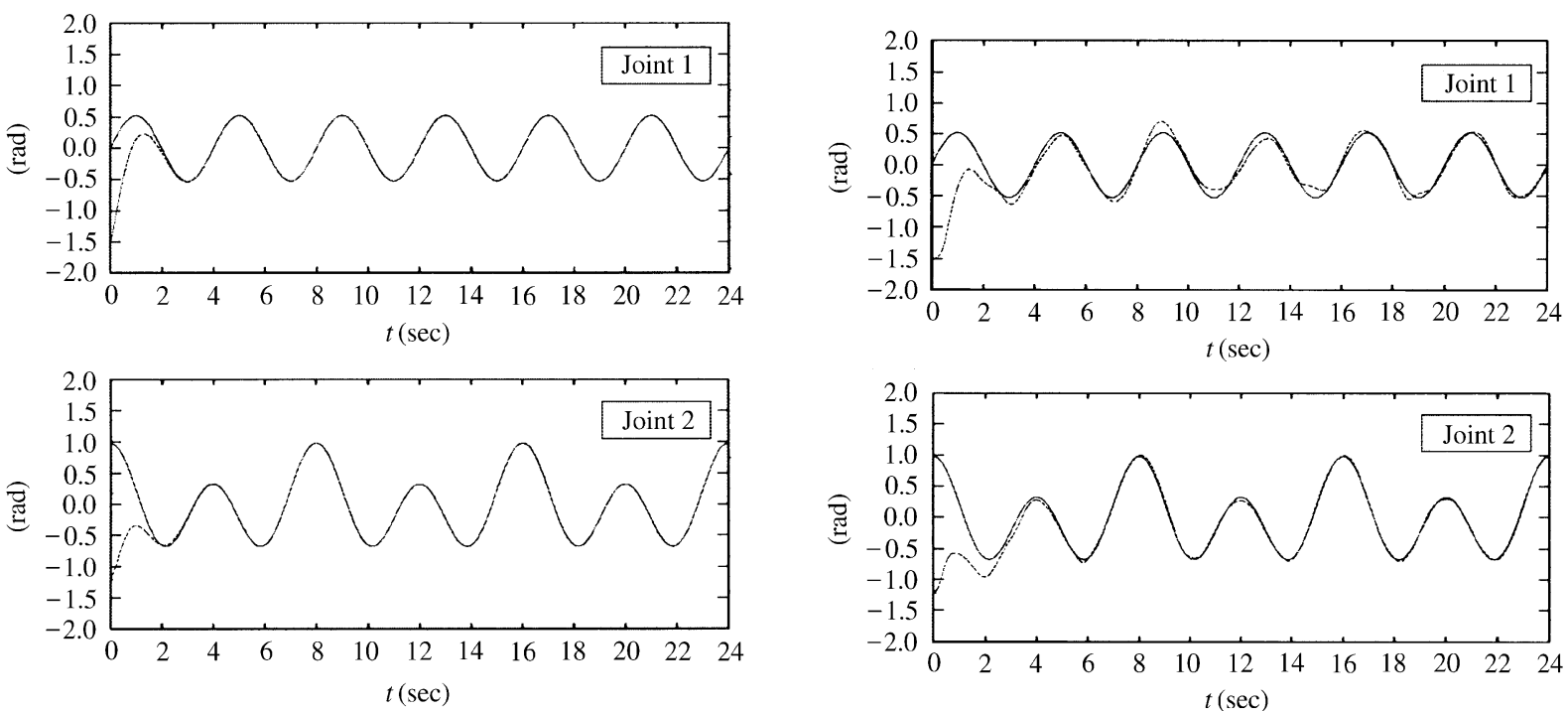

(a)

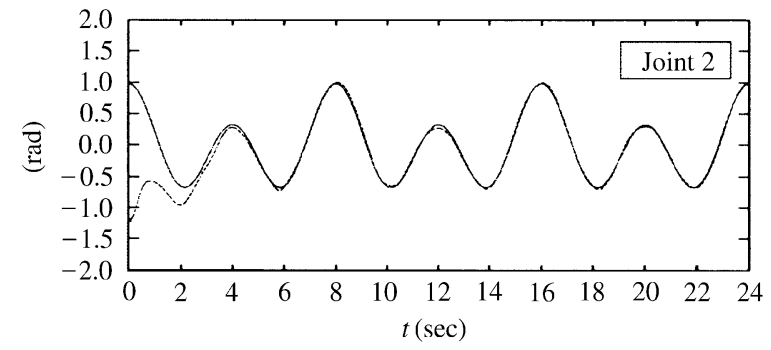

(a)
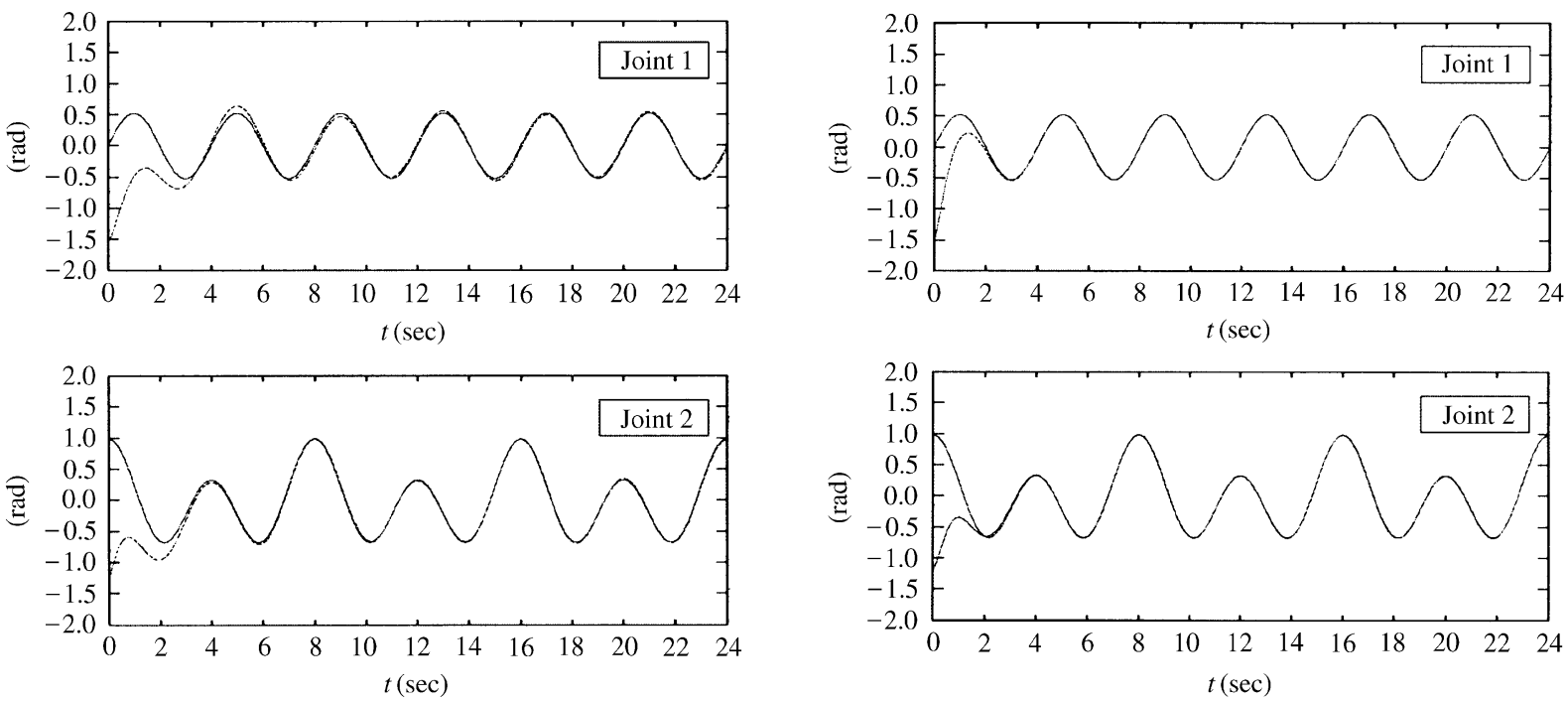

(b)

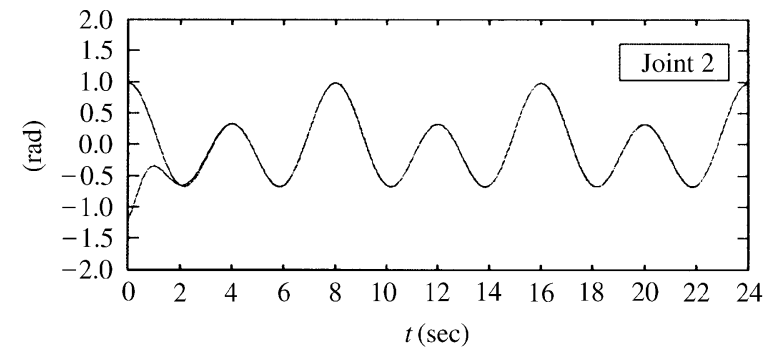

(b)

Fig. 3. Time responses of the robot control provided with the rough mathematical model and nominal parameters use (a) the gradient weight adaptive scheme, (b) the robust weight adaptive scheme.

Fig. 4. Time responses of the robot control without knowing the rough mathematical model and nominal parameters use (a) the gradient weight adaptive scheme, (b) the robust weight adaptive scheme.

\section{Appendix A}

The derivation of (7) is provided in this appendix. Referring to Fig. 5 and by (4), for the matching degree $\boldsymbol{A}^{j}$ at the left intersection point $u_{P}$ we have

$$
\boldsymbol{A}^{j}=\left(1+\left(\omega_{i i}^{j}\left(c_{i}^{j}-u_{P}\right) / a_{\mathrm{L} i}\right)\right)^{-1}
$$




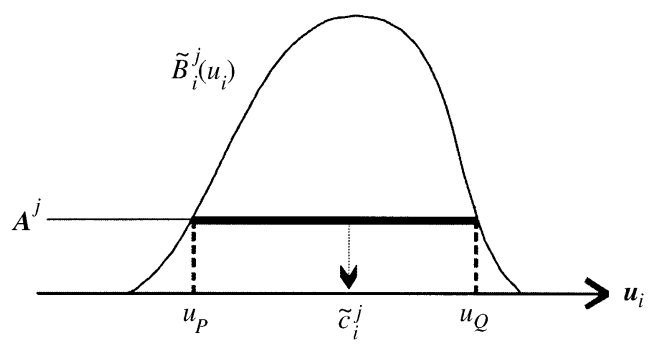

Fig. 5. The definition of $\tilde{c}_{i}^{j}$, the centroid of the line segment of height $\boldsymbol{A}^{j}$ intercepted by the fuzzy membership function.

or

$$
u_{P}=c_{i}^{j}-\frac{a_{\mathrm{L} i}}{\omega_{i i}^{j}} \sqrt{\frac{1}{\boldsymbol{A}^{j}}-1} .
$$

Similarly at the right intersection point $u_{Q}$, we have the following result:

$$
u_{Q}=c_{i}^{j}+\frac{a_{\mathrm{R} i}}{\omega_{i i}^{j}} \sqrt{\frac{1}{\boldsymbol{A}^{j}}-1} .
$$

Then by definition (6), we can obtain (7) as

$$
\tilde{c}_{i}^{j}=\left(u_{P}+u_{Q}\right) / 2
$$

\section{References}

[1] H.R. Berenji, P.S. Khefkar, Learning and tuning fuzzy logic controllers through reinforcements, IEEE Trans. Neural Networks 3 (1992) 725-740.

[2] C.S. Chen, W.L. Chen, Robust model reference adaptive control of nonlinear systems using fuzzy systems, Internat. J. System Sci. 27 (1996) 1435-1442.

[3] H.N. Farbrother, B.A. Staceyt, R. Sutton, Fuzzy self-organising control of a remotely operated submersible, IEE Internat. Conf. on Control, 1991, pp. 449-504.

[4] C.-C. Fuh, P.-C. Tung, Robust stability analysis of fuzzy control systems, Fuzzy Sets and Systems 88 (1997) $289-298$.

[5] R.A. Horn, C.R. Johnson, Matrix Analysis, Cambridge University Press, Cambridge, UK, 1985.

[6] T.A. Johansen, Stability, robustness, and performance of fuzzy model based control, Proc. 1996 IEEE Conf. Decision and Control, vol. 1, 1996, pp. 604-609.

[7] A. Kharieb, B.A. White, Robust output tracking for MIMO nonlinear systems an adaptive fuzzy systems approach, Proc. 1995 IEEE Conf. Dec. and Control, vol. 1, 1995, pp. 274-278.

[8] S. Kim, Y. Cho, M. Park, A multirule-base controller using the robust property of a fuzzy controller and its design method, IEEE Trans. Fuzzy Systems 4 (1996) 315-327.

[9] Y.F. Li, C.C. Lau, Development of fuzzy algorithms for servo systems, IEEE Control Systems Mag. (1989) 65-71.

[10] C.C. Liu, F.C. Chen, Adaptive control of non-linear continuous-time systems using neural networks - general relative degree and MIMO cases, Internat. J. Control 58 (1993) 317-335.

[11] N. Matsunaga, S. Kawaji, Hybrid controller with fuzzy and PD control, Proc. IEEE Int. Workshop Adv. Motion Control, 1990, pp. 99-106.

[12] J. Nie, D.A. Linkens, Learning control using fuzzified self-organizing radial basis function network, IEEE Trans. Fuzzy Systems 1 (1993) 280-287.

[13] R. Ordonez, J.T. Spooner, K.M. Passino, Stable multi-input multi-output adaptive fuzzy control, Proc. IEEE 1996 Conf. on Dec. and Control, vol. 1, 1996, pp. 610-615.

[14] M.M. Polycarpou, P.A. Ioannou, Robust adaptive nonlinear control design, Automatica 32 (1996) $423-427$.

[15] T.J. Procyk, E.H. Mamdani, A linguistic self-organising process controller, Automatica 15 (1979) 15-30. 
[16] M. Sugeno, Fuzzy Control, Nikan, Tokyo, Japan, 1988.

[17] L.X. Wang, Stable adaptive fuzzy control of nonlinear systems, IEEE Trans. Fuzzy Systems 1 (1993) 146-149.

[18] T. Yamazaki, E.H. Mamdani, On the performance of a rule-based self-organising controller, IEEE Conf. on Applications of Adaptive and Multivariable Control, 1982, pp. 50-55.

[19] S.Y. Yi, M.J. Chung, Robustness of fuzzy logic control for an uncertain dynamic system, IEEE Trans. Fuzzy Systems 6 (1998) 216-225.

[20] T.K. Yin, C.S.G. Lee, Fuzzy model-reference adaptive control, IEEE Trans. Systems Man Cybernet. 25 (1995) 1606-1615.

[21] B.S. Zhang, J.M. Edmunds, Self-organising fuzzy logic controller, IEE Proc. D 139 (1992) 460-464. 